\title{
Non-Intrusive Electrical Load Monitoring System Applying Neural Networks with Combined Steady-State Electrical Variables
}

\author{
Diana RACINES, John E. CANDELO, Johny MONTAÑA
}

\begin{abstract}
This paper presents a full electrical load identification model that considers steady-state parameters obtained easily from low-cost residential smart meters. The model was developed using neural networks including combinations of real power, current, impedance and admittance variables to identify the best input parameters. The monitoring model was improved by training one neural network to identify changing events and another neural network to identify the load state. The proposed model was tested using two different groups of residential loads: residential appliances measured in the laboratory and a public database of electrical measurements. The results show that the impedance model and a feedforward neural network achieved the best performance to characterise the load. In addition, when combining the different input parameters, those that consider impedance as an input parameter produced better results. The output provides simultaneous information about the operation state of all the loads before and after an event occurs.
\end{abstract}

Keywords: energy efficiency; load characterization; neural networks; non-intrusive load monitoring method; smart meters

\section{INTRODUCTION}

The modernisation of electrical networks with smart grids implies the installation of new tools and technologies throughout electrical facilities [1]. Commercial, industrial and residential users can take advantage of the enormous possibilities to interact actively with the electrical network and service providers. Some of these benefits are cost reduction, installation state monitoring, demand management, energy savings, electrical and electronic device control, price management and energy efficiency. A suitable smart installation leads to having sufficient information from the electrical installation to take appropriate actions or communicate the problem precisely.

Remote electrical load monitoring with smart devices through the electrical installation is the proper option to identify a vast number of issues in the network [2]. Traditional electrical load monitoring systems involve the use of large hardware. The main element to measure the electrical variables is an external smart meter, which identifies the general energy consumption. Other smart sensors must be installed at different circuits or electric devices to achieve the best identification of loads. However, sometimes it is impractical to install a large number of smart sensors, modify the electrical circuits and construct new facilities to measure the variables. Therefore, this suggested solution can lead to high costs of installation and maintenance [3].

Many residential users in some developing countries still have external analogue meters to measure the total energy consumption. In the future, there is a high possibility to update from analogue to smart meters. However, increasing the number of internal meters will represent a high cost for residential users and not all users will have the possibility to afford their circuit and appliance measures. Consequently, obtaining the states measures of the internal network and managing the demand will be a difficult task due to the lack of information to make the correct decision about the electricity use. A most suitable measurement method to monitor residential users must be applied for these types of residential users.

The NILM has been suggested as an alternative technique for some of the aforementioned problems [4].
The system is designed to identify the load behaviour, but to avoid measuring at some points of the electrical circuits. In this manner, the operation of each electrical device at a specific installation and the load characterisation is possible to be conducted using the general consumption curve. This system can be set up for all electrical installations, but the most suitable applications have been done for residential users. However, the researched information found in the literature has not considered event identification of groups of loads; instead, they are based on transient features that are neither useful nor affordable for most residential users. Furthermore, other possible electrical variables have not been tested for identifying appliances and the operating states.

This article presents the improvement of an electrical consumption characterisation model using the NILM. Two groups of loads were used to develop and test the NILM. The first group was formed of typical residential loads tested in laboratory conditions. The second group was selected from a database available on the web. Both groups of loads were used to determine the functioning and difference of the models and the combined variables referred on this research. The two types of loads were characterised by their operating states and power consumption. Event identification and load characterisation were performed with two separate neural networks, testing two different architectures for load characterisation. The neural networks were trained for multiple neurons in the hidden layer using the MATLAB toolbox under the training criteria previously established. Validation of the model was conducted with new data of both sets of typical loads, showing that events and operating states were identified with a high accuracy success rate for all possible changes in the load.

\subsection{Non-Intrusive Electrical Load Monitoring Method}

The NILM was created by George Hart, Ed Kern and Fred Schweppe in the 1980s with EPRI funding [5]. One of the first models was developed at M.I.T., determining the individual consumption of residential loads when they turn on and off. It was based on a detailed analysis of the steady-state current and tension of the total load [6]. 
The NILM can be produced using techniques such as probabilistic models, neural networks and support vector machines. A model is designed to continually monitor the load's behaviour and to identify possible state changes. The input values of the model can be measured by smart meters as steady state or transient variables [7]. The importance of knowing the energy consumption and operational patterns of individual electric and electronic devices in an electrical installation is related to the economic aspects that users can manage. This technique has been suggested in the literature as an option to manage users' installations, but the main requirements of this alternative are the special smart meters and algorithms.

In [8], an electrical load monitoring system was developed to identify residential devices using steady-state signals. In [9], another system was developed with the ability to disaggregate the total real power, detect the load changes and obtain specific information of the devices at residential installations. The solutions were found applying an optimisation model with only two-state devices (on or off). Results showed that this model could not detect a high amount of the total energy consumption. Another research considered real power along with specific decision rules for devices with high power consumption [10]. In this case, the accuracy to detect devices was around $80 \%$; however, the excessive training time and the need for certain specific rules for each load make it unattractive. The model proposed in [11] involved the use of real power and impedance measures obtained from an educational building to represent the consumption behaviour of loads and to identify the operation states.

Other parameters can be used to develop non-intrusive electrical load monitoring models, such as the harmonic components used in [12], obtained from the steady-state current. This variable was used as an input of a multilayer perceptron MLP and a RBF network. The recognition process of 10 electrical devices obtained success rates of $70-100 \%$.

The NILM have some common principles. First, specific features of loads must be selected and processed. After this, a hardware installation is required to detect the selected features and an algorithm is used to identify them from the total signal. Research about this topic has been more focused on the exploration of certain characteristics of the loads than on the development of algorithms [13]. Facing this, many researchers have begun to publish their databases to facilitate the work of other researchers and promote the development of algorithms as in [14:17].

Most non-intrusive electrical load monitoring models are focused on identifying a load or group of loads, but they usually do not consider event identification. This makes it harder on the implementation of such models in a real installation. Furthermore, many techniques are developed based on transient features that require the use of an advanced meter to measure electrical variables. These meters are expensive and cannot be afforded by all endusers.

Models based on steady-state electrical parameters for the identification of load states in a non-intrusive way usually use real power $[8,18]$, reactive power $[6,19]$, voltage [20, 21], current [16] and harmonics [12]. However, the impedance and admittance parameters are practically unused. Furthermore, these models usually do not consider the combination of parameters to determine the most suitable to perform the non-intrusive electrical load identification.

\section{MATERIALS AND METHODS}

The general methodology implemented for the development and validation of the proposed non-intrusive electrical load monitoring model is shown in Fig. 1, where the letter I refers to input data for developing the model, the letter $\mathrm{P}$ is the process where the neural network is designed and trained, and the $\mathrm{O}$ is the output data of the methodology.

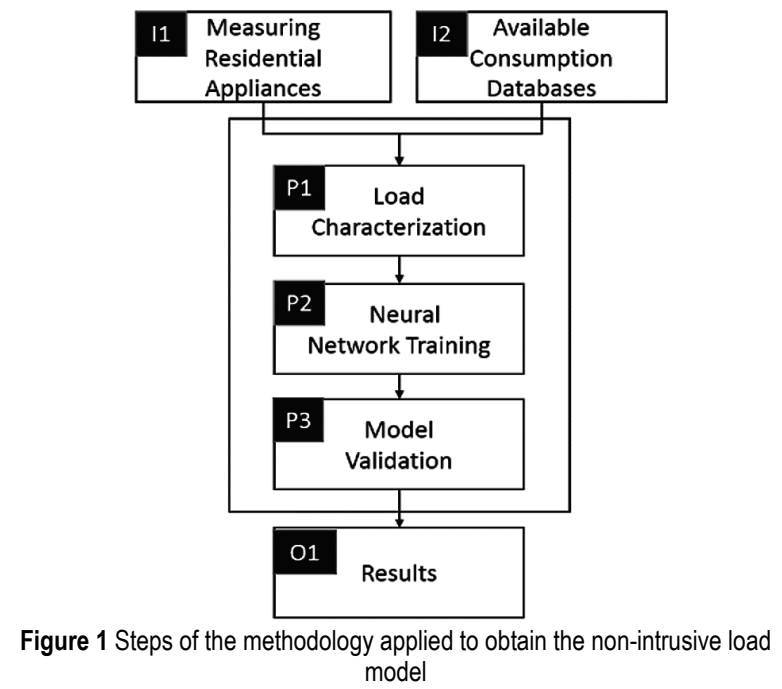

We selected as input data two types of load: (I1) electrical variables of appliances normally used for residential users and (I2) the consumption data available in the internet databases. Next, we process data and characterise the load (P1). Then, we trained two neural networks to identify the load and the operation states of the different appliances in the databases (P2). Following, we validate the model with new data in the databases to identify the errors (P3). Finally, we obtained the results that are presented in Section 4.

\subsection{Measuring Residential Appliances (I1)}

Loads with two operating states, on and off, which are most commonly found in a residential installation, were selected for this group. For the test, four devices typically found in residential loads were selected as part of the experiment and their nominal power consumption set as follows: an iron with a consumption of $1200 \mathrm{~W}$, a blender with a consumption of $240 \mathrm{~W}$, a sandwich maker with a consumption of $660 \mathrm{~W}$ and a fan with a consumption of $140 \mathrm{~W}$. These electrical loads were selected because they are commonly used in every home, they have a range of energy consumption and they represent both resistance (the iron and the sandwich maker) and inductance (the fan and the blender).

A residential smart meter was used to register the following electrical parameters: voltage, current, real power, reactive power, apparent power, frequency and power factor. Individual loads and combinations of loads were measured to obtain all possible changes in power 
consumption and the behaviour of electrical signals. According to the operation state, there are 80 possible combinations of load changes considering four types of events: a Type 1 events refers to a change in the operating state of just one load; a Type 2 event involves a change in the operating state of two loads at the same time; in Type 3 events, three loads change their operating state at the same time; and Type 4 events, where all the loads change their operating state at the same time. All types of events and their combinations were tested in this study.

The tag associated to each load (A, B, C or D) and their operating states (1-8), are shown in Tab. 1. The odd state of each load represents that the load is off while the even state represents the load is on. Load states are named consecutively from 1 to 8 as it was identified that this minimises the confusion of the neural network in load identification.

Table 1 First group of loads
\begin{tabular}{|l|c|c|}
\hline \multicolumn{1}{|c|}{ Load } & Tag & States \\
\hline Iron & A & 1,2 \\
\hline Blender & B & 3,4 \\
\hline Sandwich maker & C & 5,6 \\
\hline Fan & D & 7,8 \\
\hline
\end{tabular}

\subsection{Available Consumption Databases (12)}

Measurements made over a real home registered on the AMPds dataset [16] were used for this group of loads. This database was selected mainly due to the high number of electrical parameters recorded. Of the 19 circuits available to the database, some are composed of a single load and others of multiple loads. This database consists of measurements of 11 electrical parameters made over a real home at Vancouver, Canada, for one-year period. For each electrical circuit of the home and the total electrical consumption, the voltage, current, frequency, displacement power factor, apparent power factor, real power, real energy, reactive power, reactive energy, apparent power and apparent energy were measured and recorded. The first version of the AMPds was released in 2013.

Two circuits of each type (single load and multiple loads) were selected by their influence on total energy consumption. This was done through a correlation analysis between the power consumed by each circuit and the total power of the house Thus, circuits for the heat pump (HPE), dishwasher (DWE), basement plugs and lights (BME), and TV/PVR/AMP (TVE) were selected to be identified by the model, because they had the higher correlation coefficients.

\begin{tabular}{|c|c|c|c|}
\hline Load & Tag & States & Power Range (W) \\
\hline \multirow{2}{*}{ Heat pump } & \multirow{2}{*}{ HPE } & 1 & $0-50$ \\
\hline & & 2 & $51-3030$ \\
\hline \multirow{3}{*}{$\begin{array}{c}\text { Basement } \\
\text { plugs and lights }\end{array}$} & \multirow{3}{*}{ BME } & 3 & $0-45$ \\
\hline & & 4 & $46-150$ \\
\hline & & 5 & $151-1570$ \\
\hline \multirow{5}{*}{$\begin{array}{c}\text { TV/PVR/AMP } \\
\text { Dishwasher }\end{array}$} & \multirow{5}{*}{$\begin{array}{l}\text { TVE } \\
\text { DWE }\end{array}$} & 6 & $0-66$ \\
\hline & & 7 & $67-460$ \\
\hline & & 8 & $0-30$ \\
\hline & & 9 & $31-190$ \\
\hline & & 10 & $191-805$ \\
\hline
\end{tabular}

Analysing the load profile of these loads, it was discovered that loads HPE and TVE can be modelled with two operation states (low and high), whereas loads BME and DWE can be modelled with three operation states (low, medium and high). Therefore, Tab. 2 shows the loads of the second group, their tags and their operation states. The power range of each state was established by the observation of the power profile obtained for each load.

\subsection{Load Characterisation (P1)}

Two groups of typical residential loads were selected. To characterise all the loads, measurements of their electrical parameters were obtained for all their operating conditions. This brought the proposed model to be based on manual setup (MS-NILM) [6], which implies an initial degree of intrusion. All the loads were characterised by identifying their operating states and determining the behaviour of the electrical parameters for each of them. This characterisation allows to determine when an event occurs, defining an event as a change in the operating state of one or more loads.

\subsection{Neural Network Training (P2)}

The general structure of the proposed model is shown in Fig. 2. The neural networks involved in the model were designed in terms of the quantity and type of inputs, number of hidden layers, number of neurons in the hidden layer, number of outputs and type of architecture. After this, the training algorithm is selected and performed with the previously collected data. The block diagram shows that the total consumption curve must be obtained. This is the sum of the energy consumption of all the installation loads. This figure shows that we used two ANNs for the event identification and the state identification.

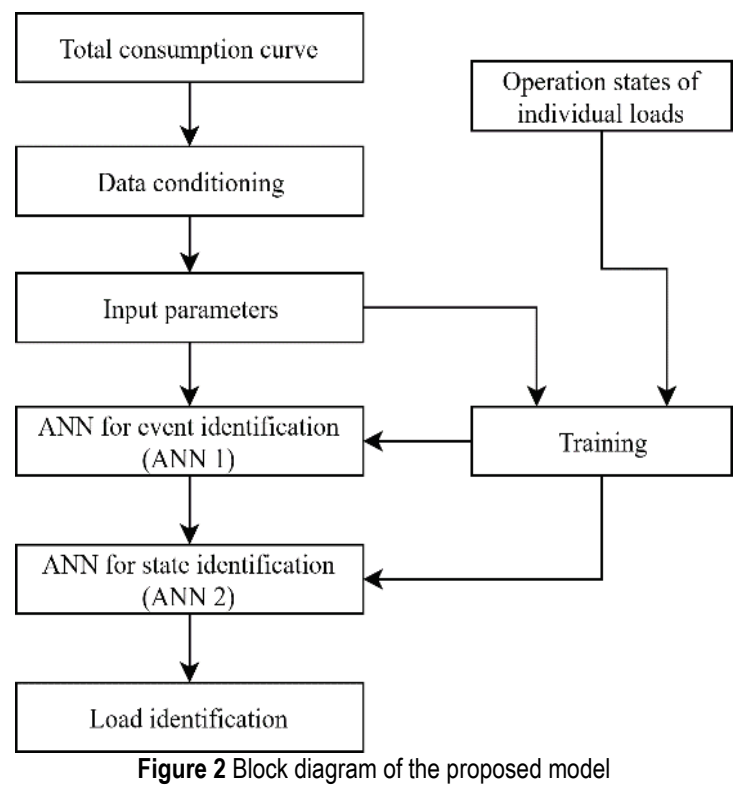

Considering $n$ loads, when load $i$ has a power change between time $t$ and time $t-1$, a delta value $\left(\mathrm{d} P_{i}\right)$ can be calculated. The total curve has significant changes when the electrical devices change their operation state. These changes are very significant, that is why every moment the behaviour of the total curve is analysed so that the event identification can be determined. 
These changes in the power consumption of the loads are reflected in the total power consumption according to Eq. (1). The term $P_{t}(t)$ is the total power consumption and the term $P_{i}(t)$ is the power consumption of load $i$ :

$$
P_{t}(t)=\sum_{i=1}^{n} P_{i}(t)
$$

When the total delta power $\mathrm{d} P_{t}$ exceeds a certain minimum value, it might indicate that an event has occurred, which implies that one or more loads have changed their operation state. This method of identifying an event is very simple, although it can be ineffective, especially when there are loads that have variable power consumption even though they are on the same operation state. Facing the previous inconvenience, the present article proposes the use of an artificial neural network for event identification (ANN 1). Once an event has been identified, it must be determined which devices switched their operation state by using another neural network (ANN 2). Thus, it will be possible to know, at every moment, which devices have more impact on the installation's total electric power consumption.

According to the load data analysed, it was found that the delta minimum total power is $19 \mathrm{~W}$ both for the first and second groups of loads. Fig. 3 shows the flowchart of the proposed model for the evaluation of a total consumption curve. It is observed that the input parameters of ANN 2 can be selected since this network was trained with different combinations of electrical parameters.

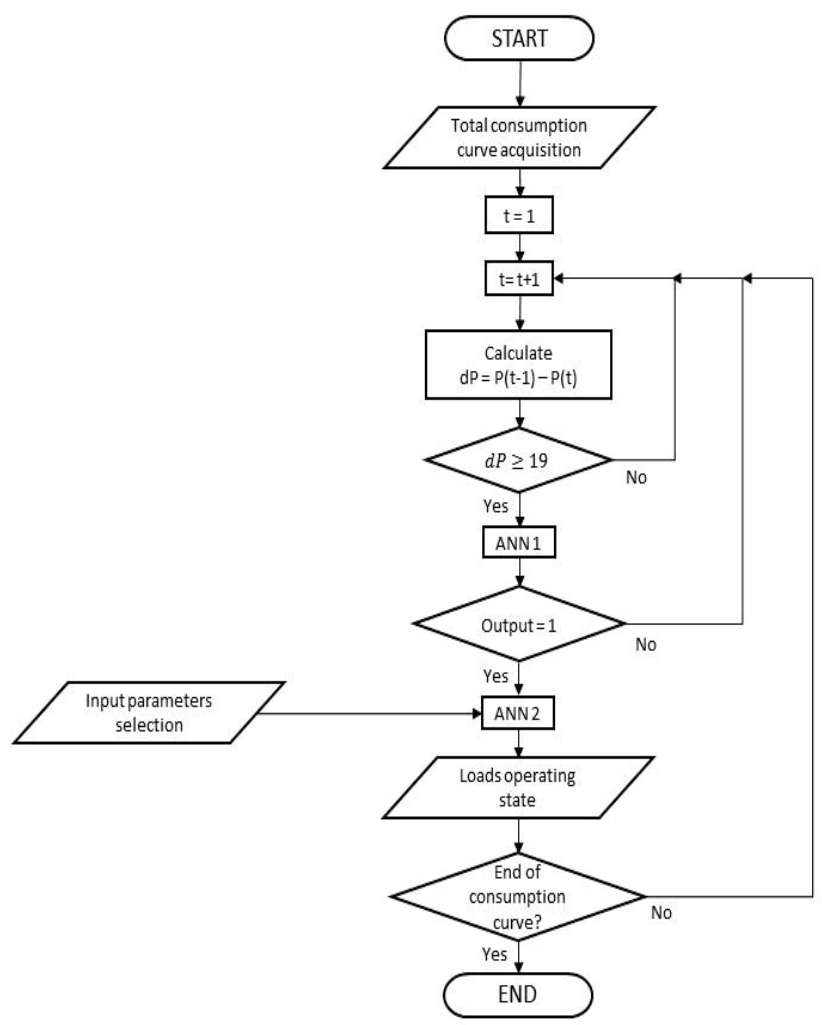

Figure 3 Flowchart of the proposed model

ANN for event identification (ANN 1). When a possible event is identified in the total power curve, the neural network for event identification is applied. The input of this network is the value of the total real power during and instantly before a possible event occurs. The output can take the value 1 or -1 , indicating that an event occurs or not, respectively. This described structure is shown in Fig. 4. ANN 1 is a multilayer feedforward network.

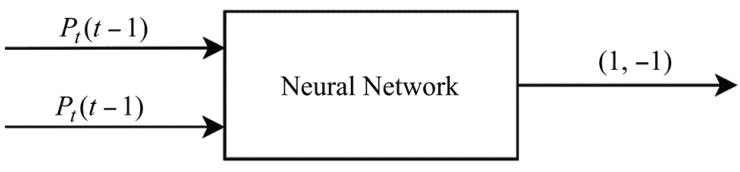

Figure 4 Structure of neural network for event identification

ANN for state identification (ANN 2). Once an event has been identified, the information is passed to ANN 2 to determine which load or loads switched their operating state. Two different architectures are used for ANN 2: a multilayer feed forward back propagation network and an Elman network. This is done to determine the behaviour of networks with and without feedback in non-intrusive load monitoring models. All neural networks developed have one hidden layer. The proposed model considers as input parameters steady-state features due to its intended use as an economical and practical tool for residential end-users who typically do not have meters at their installations that optimally measure transient features. In search of electrical parameters that effectively allow the development of nonintrusive load monitoring models, six sets of input parameters are proposed that involve the use of real power, current, admittance and impedance. Fig. 5 shows the sets of input parameters proposed and the structure of the neural network for state identification; where, $P$ is the real power, $Z$ is the impedance, $Y$ is the admittance, and $I$ is the current.

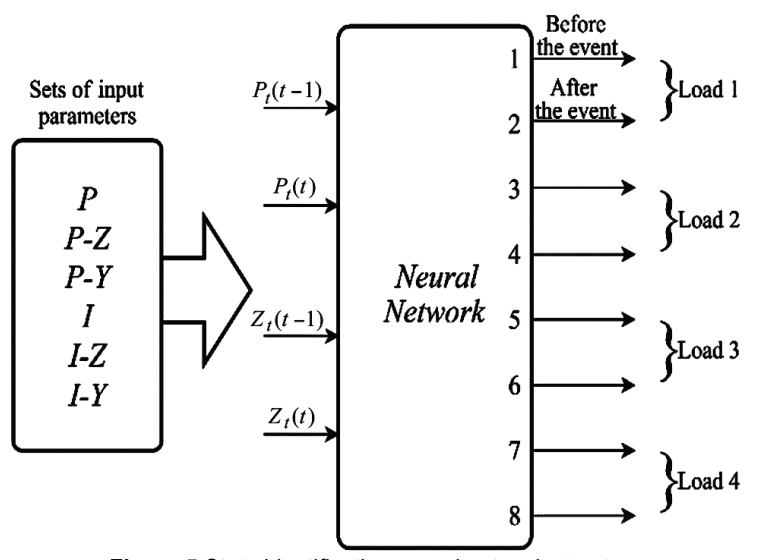

Figure 5 State identification neural network structure

ANN 2 has two or four inputs, depending on the selected set, and eight outputs. The inputs are the values of the electrical parameters selected at the instant an event occurs $(t)$ and at the instant before $(t-1)$. The outputs correspond to the operation state of each device before and after the manifestation of the event. Outputs 1 and 2 are associated with loads 1, 3 and 4 with load 2, 5 and 6 with load 3, 7 and 8 with load 4. Thus, odd outputs of each device correspond to its operating state before the event and the even outputs of the state after the event.

The outputs of the neural network can take full values between 1 and 8 for the first group of loads, and between 1 and 10 for the second group. Each of these values represents a specific operation state as previously established. Input parameters of the networks are normalised in a range between 0 and 1 , using a $\min / \max$ 
lineal normalisation [22] in order to improve the learning process. Thus, the original value $x_{k}$ takes a new value $x_{k}^{*}$, with Eq. (2). In this equation, $x_{1}^{\min }$ and $x_{1}^{\max }$ are the minimum and maximum values of the set of input data, respectively:

$$
x_{k}^{*}=\left[\frac{x_{k}-x_{1}^{\min }}{x_{1}^{\max }-x_{1}^{\min }}\right] \text {. }
$$

Regarding impedance, in the case when all the loads are off and, therefore, the impedance is infinite, it is assumed an input value of 10 , which is higher than the standardised upper limit.

Training neural networks. The training of both ANN 1 and ANN 2 was performed through the neural network toolbox of MATLAB software. The Levenberg-Marquardt learning algorithm, tansig transfer function in the hidden layer and linear transfer function in the output layer were used as recommended for the kinds of networks employed [23]. Levenberg-Marquardt is a second-order algorithm and ranked as one of the most efficient training algorithms for small- and medium-sized patterns [24]. It helps decrease the number of neurons needed to fulfil a task, thereby improving the generalization ability of the neural network [25].

To establish the number of neurons that the hidden layer must have, several training cases were performed. For the event identification network, the number of hidden neurons varied between 5 and 50, whereas for the state identification network it varied between 10 and 90 . Seventy percent of the input dataset was used for the network training, $15 \%$ for the validation and $15 \%$ for the testing. For each of these stages, it was registered the value of the mean squared error (MSE), the number of events where the network made a wrong classification and the success percentage obtained.

As a result of this stage, it was established that the best results for the event identification network were obtained with 20 and 50 neurons in the hidden layer. Further details are given in the following sections.

Other neural networks, similar to those proposed in this paper, have been used to solve problems related to electrical load identification. In [26], Multi-Layer Feed Forward networks were used to identify load patterns by using one network for each electrical load. The authors in [27] also used a Multi-Layer Feed Forward network based on the back propagation method (due to its capacity to identify the similarity between given data and known data) to identify three loads (two induction motors and a bank of loads supplied by a thyristor rectifier), using as input the real power, the reactive power and the turn-on transient energy. Another study used as input the harmonic spectrum at the service point and harmonic content table of nine typical electrical loads [28] for a Multi-Layer Feed Forward neural network. This network was trained using the Levenberg-Marquardt algorithm while the sigmoid transfer function was used for the hidden layer and a linear transfer function for the output layer. The average error obtained was $10.06 \%$.

\subsection{Model Validation (P3)}

The validation of the non-intrusive load monitoring model was performed with a new set of consumption data, different to that employed for training the neural networks. The validation was performed for both groups of loads: the first one corresponds to the loads measured in the laboratory and the other one is information obtained from the consumption database.

First, the performances of the event identification network and the state identification network were studied separately and before working as coupled networks. Then, the performance of the complete model was analysed, which implied studying the errors associated with the coupled networks as the input of the state identification network depends on the output of the event identification network.

\subsection{Output Results (01)}

In this step, we analysed all the results obtained from the experiment to identify the best neural network for load identification and the best input variables used. The results are presented for both types of loads used as input in the process. We analysed the results obtained from the neural network training and the validation of the load data used as input to identify the success of the used methods.

\section{RESULTS AND ANALYSIS}

The aim of this section is to show how the nonintrusive load monitoring model based on combined electrical variable can identify different appliances of the load and their states.

\subsection{First Group of Loads}

The results included for this type of load are the neural network training and the validation of the model obtained for different data included in the database.

Neural network training. To train the artificial neural networks, an energy consumption curve was created based on the measurements performed over the loads. This representative curve includes 780 possible events and 618 real events. For training ANN 1, the number of hidden neurons varied between 5 and 50 by increments of 5 . In this case, a success percentage between $98.21 \%$ and $98.85 \%$ was obtained. For the training of ANN 2, the number of hidden neurons varied between 10 and 90 for the feedforward network, and between 10 and 65 for the Elman network.

Table 3 Better success percentage feedforward network vs Elman network first group of loads

\begin{tabular}{|c|c|c|}
\hline \multirow{2}{*}{$\begin{array}{c}\text { Input } \\
\text { Parameters }\end{array}$} & \multicolumn{2}{|c|}{ No. of Cases with Better Success Percentage } \\
\cline { 2 - 3 } & Feedforward & Elman \\
\hline$P$ & 6 & 3 \\
\hline$P-Z$ & 6 & 3 \\
\hline$P-Y$ & 7 & 2 \\
\hline$I$ & 7 & 2 \\
\hline$I-Z$ & 9 & 0 \\
\hline$I-Y$ & 7 & 2 \\
\hline
\end{tabular}


In general, the training time of the Elman network is considerably higher than the training time of the feedforward network. Results also showed that in most cases, the feedforward network produces a higher success percentage than the Elman network. This can be observed in Tab. 3, where for all sets of input parameters it is shown in how many cases it was better for each network.

Due to the better performance of the feedforward network, it is most interesting to analyse the results obtained with each set of input parameters with this kind of network. In this sense, Figs. 6 and 7 show the success percentage obtained for the input sets that use real power and for the input sets that use current, respectively. In these figures, the $x$-axis represents the number of neurons in the hidden layer. It is observed that the best results correspond to the input sets that use impedance $(P-Z$ and $I-Z)$ since the success percentage obtained with this parameter is the highest for most cases.

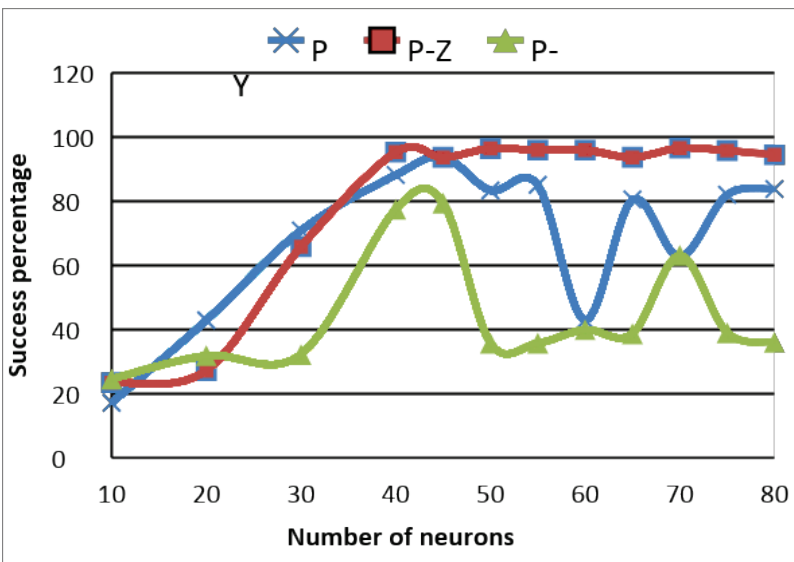

Figure 6 Feedforward network success percentage for $P, P-Z$ and $P-Y$ parameters: first group of loads

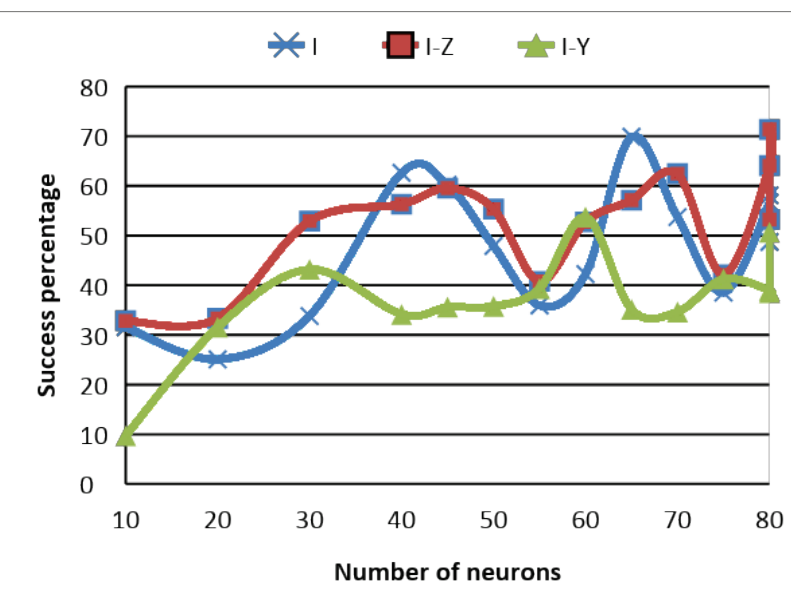

Figure 7 Feedforward network success percentage for $I, I-Z$ and $I-Y$ parameters: first group of loads

Table 4 Best event identification network results - first group of loads

\begin{tabular}{|c|c|c|}
\hline \multirow{2}{*}{} & \multicolumn{2}{|c|}{20 Neurons } \\
\cline { 2 - 3 } & Training & Validation \\
\hline No. of Possible Events & 780 & 119 \\
\hline No. of Errors & 9 & 2 \\
\hline Success (\%) & 98.85 & 98.32 \\
\hline
\end{tabular}

Neural network validation. The validation set includes 119 possible events and 111 real events. The possible events were applied to the identification networktrained load, obtaining that the network with 20 neurons on the hidden layer delivers the best results for both the training and the validation. Therefore, this network is selected for event identification. Results obtained for training and validation are shown in Tab. 4 .

The detailed behaviour of event identification is shown in the confusion matrix of Tab. 5. This matrix indicates the correlation between the model outputs and what the target must achieve. In this case, the model indicates one false event (false positive) and fails to identify one event (false negative).

Table 5 Confusion matrix of event identification - first group of loads

\begin{tabular}{|c|c|c|c|}
\hline \multicolumn{2}{|c|}{} & \multicolumn{2}{|c|}{ Model Output } \\
\cline { 3 - 4 } & Event (1) & No Event $(-1)$ \\
\hline Real & Event & 110 & 1 \\
\hline target & No event $(-1)$ & 1 & 7 \\
\hline
\end{tabular}

With the values of the confusion matrix identified, it is possible to calculate two indicators for event identification: Precision and Recall. Precision expresses the proportion of the predicted events that correspond to real events while Recall indicates the proportion of the real events that were properly identified. These indicators are calculated as shown in Eqs. (3) and (4), where $T P=$ true positive, $F P=$ false positive and $F N=$ false negative:

$$
\begin{aligned}
& \text { Precision }=\frac{T P}{T P+F P}=\frac{110}{110+1}=0.9909, \\
& \text { Recall }=\frac{T P}{T P+F N}=\frac{110}{110+1}=0.9909 .
\end{aligned}
$$

Precision and Recall indicate good event identification by the model considering that their optimum value is 1 .

Regarding ANN 2, the 111 real events of the validation set were applied to the trained feedforward networks. For each set of input parameters, the network with the best performance during training and validation was selected. These results, displayed in Tab. 6, indicate that the highest success percentage was obtained using the $P Z$ input parameter.

\begin{tabular}{|c|c|c|c|c|}
\hline \multicolumn{2}{|c|}{ Input Parameters } & $\begin{array}{l}\text { No. of } \\
\text { Events }\end{array}$ & $\begin{array}{l}\text { No. of } \\
\text { Errors }\end{array}$ & $\begin{array}{c}\text { Success } \\
(\%)\end{array}$ \\
\hline \multirow{2}{*}{$\begin{array}{c}P \\
\text { (45 neurons) }\end{array}$} & Training & 618 & 38 & 93.85 \\
\hline & Validation & 111 & 8 & 92.79 \\
\hline \multirow{2}{*}{$\begin{array}{c}P Z \\
\text { (50 neurons) }\end{array}$} & Training & 618 & 22 & 96.44 \\
\hline & Validation & 111 & 6 & 94.59 \\
\hline \multirow{2}{*}{$\begin{array}{c}P Y \\
\text { (45 neurons) }\end{array}$} & Training & 618 & 127 & 79.45 \\
\hline & Validation & 111 & 26 & 76.57 \\
\hline \multirow{2}{*}{$\begin{array}{c}I \\
\text { (65 neurons) }\end{array}$} & Training & 618 & 186 & 69.90 \\
\hline & Validation & 111 & 30 & 72.97 \\
\hline \multirow{2}{*}{$\begin{array}{c}I Z \\
\text { (85 neurons) }\end{array}$} & Training & 618 & 177 & 71.36 \\
\hline & Validation & 111 & 38 & 65.77 \\
\hline \multirow{2}{*}{$\begin{array}{c}I Y \\
\text { (60 neurons) }\end{array}$} & Training & 618 & 286 & 53.72 \\
\hline & Validation & 111 & 60 & 45.95 \\
\hline
\end{tabular}

Table 6 Results of best state identification networks - first group of loads

Applying the validation set to the complete model, by coupling ANN 1 and ANN 2, it was obtained that 105 events were properly classified, 5 events were wrongly classified and 1 event was not identified. This presumes that the model made six mistakes and had a $94.59 \%$ success rate. Tab. 7 shows the confusion matrix for the state identification made by the model during the validation and 
Fig. 8 shows the error rate of the model on the identification of each load.

Table 7 Model state identification confusion matrix - first group of loads

\begin{tabular}{|c|c|c|c|c|c|c|c|c|c|}
\hline & \multicolumn{8}{|c|}{ Model Output } \\
\hline & & 1 & 2 & 3 & 4 & 5 & 6 & 7 & 8 \\
\hline \multirow{8}{*}{ 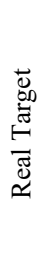 } & 1 & 106 & 0 & 0 & 0 & 0 & 0 & 0 & 0 \\
\hline & 2 & 2 & 114 & 0 & 0 & 0 & 0 & 0 & 0 \\
\hline & 3 & 0 & 0 & 120 & 2 & 0 & 0 & 0 & 0 \\
\hline & 4 & 0 & 0 & 2 & 98 & 0 & 0 & 0 & 0 \\
\hline & 5 & 0 & 0 & 0 & 0 & 108 & 2 & 0 & 0 \\
\hline & 6 & 0 & 0 & 0 & 0 & 0 & 112 & 0 & 0 \\
\hline & 7 & 0 & 0 & 0 & 0 & 0 & 0 & 115 & 2 \\
\hline & 8 & 0 & 0 & 0 & 0 & 0 & 0 & 2 & 103 \\
\hline
\end{tabular}

From the confusion matrix, it is observed that the differences between the model output and the real output occurred on states of the same load. This means that the model did not confuse any load with another. It is also remarked that the error amount is low. This is emphasised in Fig. 8, where the error rate in all loads is lower than $5 \%$. Loads B (blender) and D (fan) had a higher error rate, which can be explained by the fact that these loads are powered by an engine.

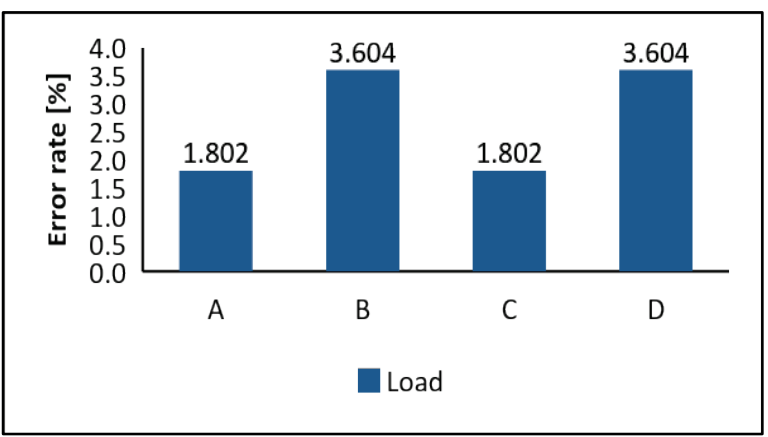

Figure 8 Load errors in model validation: first group of loads

\subsection{Second Group of Loads}

The results included for this type of load are the neural network training and the validations of the model obtained for different data included in the database.

Table 8 Better success percentage feedforward network vs Elman network second group of loads

\begin{tabular}{|c|c|c|}
\hline \multirow{2}{*}{$\begin{array}{c}\text { Input } \\
\text { Parameters }\end{array}$} & \multicolumn{2}{|c|}{ No. of Cases with Better Success Percentage } \\
\cline { 2 - 3 } & Feedforward & Elman \\
\hline$P$ & 1 & 4 \\
\hline$P-Z$ & 3 & 1 \\
\hline$P-Y$ & 3 & 2 \\
\hline$I$ & 3 & 2 \\
\hline$I-Z$ & 3 & 2 \\
\hline$I-Y$ & 3 & 2 \\
\hline
\end{tabular}

Neural network training. The training was performed using three months of available data, which provided enough information about the consumption behaviour of the loads and their changes of operation states. During this time, 10,520 possible events occurred, of which 2,641 corresponded to real events. For the training of ANN 1, the numbers of hidden neurons vary in the same way described in Section 4.1. A success percentage between $95.95 \%$ and $97.89 \%$ was obtained. For the training of ANN 2, the number of hidden neurons varied between 10 and 80 for the feedforward network and between 10 and 45 for the Elman network. This is because the computational effort required for the Elman network is too high above 45 neurons in the hidden layer. Tab. 8 shows that in most cases, the feedforward network produces a higher success percentage than the Elman's network. Besides, the training time of the Elman's network is considerably higher than the training time of the feedforward network.

Due to the better performance of the feedforward network, it is worth analysing the results obtained for each set of input parameters. In this sense, Figs. 9 and 10 show the success percentages obtained for the input sets that use real power and for the input sets that use current, respectively. It is observed again that the best results correspond to the input sets that use impedance $(P-Z$ and $I-$ $Z$ ) since the success percentage obtained with this parameter is the highest for most cases.

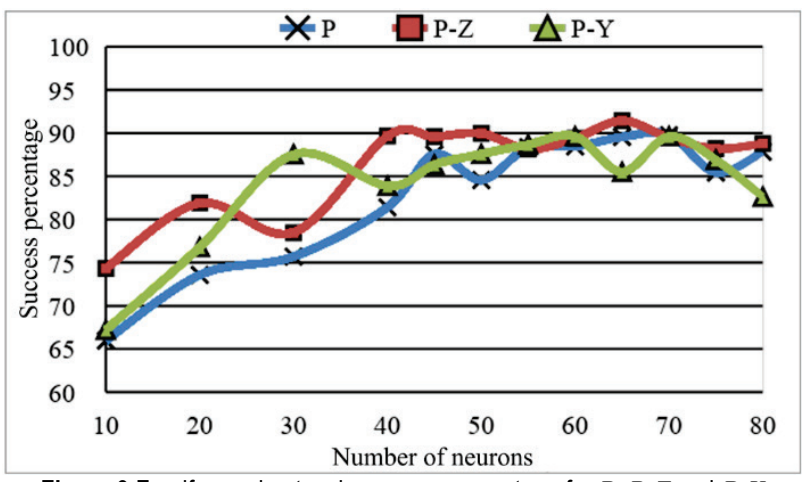

Figure 9 Feedforward network success percentage for $P, P-Z$ and $P-Y$ parameters: second group of loads

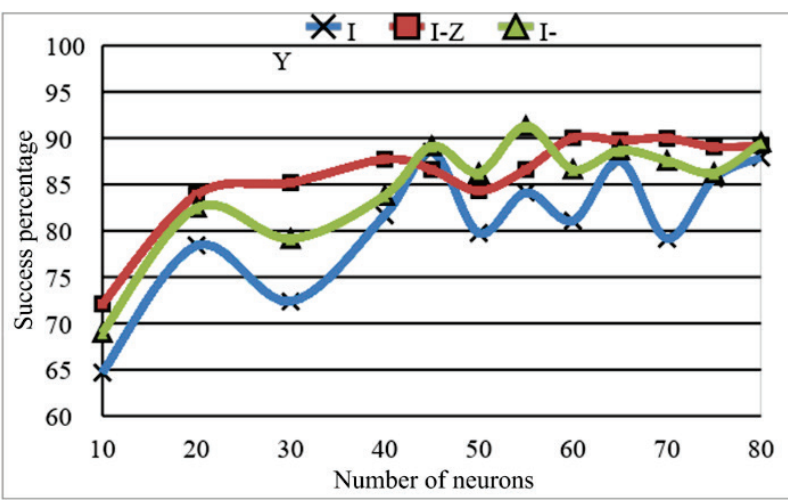

Figure 10 Feedforward network success percentage for $I, I-Z$ and $I-Y$ parameters: second group of loads

Table 9 Results of the event identification network - second group of loads

\begin{tabular}{|c|c|c|}
\hline \multirow{2}{*}{} & \multicolumn{2}{|c|}{40 Neurons } \\
\cline { 2 - 3 } & Training & Validation \\
\hline No. of possible events & 10,520 & 1,523 \\
\hline No. of errors & 222 & 30 \\
\hline Success (\%) & 97.89 & 98.03 \\
\hline
\end{tabular}

Neural network validation. For the second group of loads, the validation curve consists of one week of data measurements different to the weeks used for the training. This curve includes 1,523 possible events, from which 340 refer to real events. The possible events were applied to the trained load identification networks, obtaining that the network with 40 neurons on the hidden layer gave the best results for both training and validation. Therefore, this network is selected for event identification. Results obtained for training and validation are shown in Tab. 9. 
The detailed behaviour of event identification is shown in the confusion matrix of Tab. 10. In this case, the model indicates 10 false events (false positives) and fails to identify 20 events (false negatives).

Table 10 Event identification confusion matrix - second group of loads

\begin{tabular}{|c|c|c|c|}
\hline & \multicolumn{2}{|c|}{ Model Output } \\
\hline & & Event (1) & No Event $(-1)$ \\
\hline \multirow{2}{*}{ Real target } & Event (1) & 320 & 20 \\
\hline & No event $(-1)$ & 10 & 1,173 \\
\hline
\end{tabular}

With the confusion matrix, Precision and Recall values were calculated using Eqs. (5) and (6). Results indicate good event identification by the model.

$$
\begin{aligned}
& \text { Precision }=\frac{T P}{T P+F P}=\frac{320}{320+10}=0.9697 \\
& \text { Recall }=\frac{T P}{T P+F N}=\frac{320}{320+20}=0.9411 .
\end{aligned}
$$

Regarding ANN 2, the 340 real events of the validation set were applied to the trained feedforward networks. For each set of input parameters, the network with the best performance during training and validation was selected.

The results shown in Tab. 11 again indicate that the highest success percentage was obtained using the $\mathrm{PZ}$ input parameter.

Table 11 Results of the state identification networks - second group of loads

\begin{tabular}{|c|c|c|c|c|}
\hline \multicolumn{2}{|c|}{ Input Parameters } & $\begin{array}{c}\text { No. of } \\
\text { Events }\end{array}$ & $\begin{array}{c}\text { No. } \\
\text { of Errors }\end{array}$ & $\begin{array}{c}\text { Success } \\
(\%)\end{array}$ \\
\hline \multirow{2}{*}{$\begin{array}{c}\text { P } \\
\text { (45 neurons) }\end{array}$} & Training & 2,641 & 270 & 89.78 \\
\cline { 2 - 5 } & Validation & 340 & 75 & 77.94 \\
\hline $\begin{array}{c}\text { PZ } \\
\text { (50 neurons) }\end{array}$ & Training & 2,641 & 226 & 91.44 \\
\cline { 2 - 5 } & Validation & 340 & 57 & 83.23 \\
\hline $\begin{array}{c}\text { PY } \\
\text { (45 neurons) }\end{array}$ & Training & 2,641 & 274 & 89.63 \\
\cline { 2 - 5 } & Validation & 340 & 72 & 78.82 \\
\hline $\begin{array}{c}\text { I } \\
\text { (65 neurons) }\end{array}$ & Training & 2,641 & 319 & 87.92 \\
\cline { 2 - 5 }$(85$ neurons) & Validation & 340 & 66 & 80.59 \\
\cline { 2 - 5 } & Training & 2,641 & 264 & 90.00 \\
\hline \multirow{2}{*}{$\begin{array}{c}\text { IY } \\
\text { (60 neurons) }\end{array}$} & Validation & 340 & 65 & 80.88 \\
\cline { 2 - 5 } & Training & 2,641 & 229 & 91.33 \\
\hline
\end{tabular}

Applying the validation set to the complete model by coupling ANN 1 and ANN 2, it was obtained that 275 events were properly classified, 45 events were wrongly classified and 20 events were not identified. This translates that the model made 65 errors $(80.88 \%$ accuracy). The reduction in success percentage compared with that obtained for the first group of loads in Section 5.1 is because the second group uses circuits containing various types of loads, loads with a higher number of operation states and more variable energy consumption.

The state identification confusion matrix made by the model during the validation is shown in Tab. 12 and the error rates of the model on the identification of each load are presented in Fig. 11.

The confusion matrix shows that there are some cases where the operating states of a load are confused with those of another load, except for load HPE (outputs 1 and 2). However, the error rate is lower than $2 \%$ for every load; thus, we can conclude that the model distinguishes different types of loads. This figure shows that load HPE has the lower error rate. This is because on this load, the power consumption of each state varies in a small range, whereas the other loads have higher variability. Furthermore, circuits conformed by more than one load (BME and TVE) had the higher error rate.

Table 12 Results of the state identification confusion matrix model - second group of loads

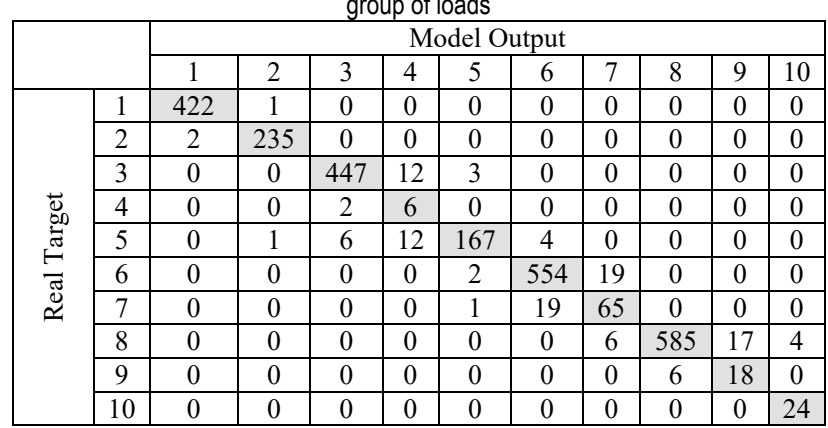

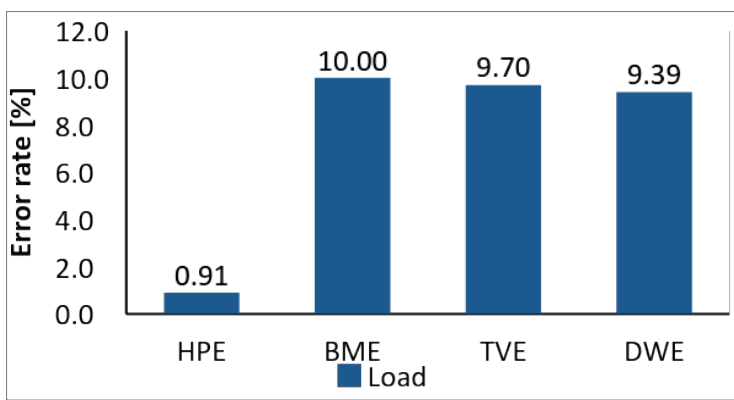

Figure 11 Load errors in model validation: second group of loads

\section{CONCLUSIONS}

This paper presented a non-intrusive load monitoring model based on neural networks trained with mixed variables measured using low-cost smart meters. The method was applied for residential loads aiming to identify the events and the state of loads. Results showed that all events and the states of load operation were identified with high accuracy for both individual loads and circuits consisting of various loads before and after the events. Two neural networks were used-one for event identification and the other for state identification - to identify the events from the total energy consumption and establish the load operation states after events. The model performed nonintrusive monitoring regardless of the number of loads that change their operation state simultaneously; in addition, it showed high accuracy while distinguishing one load from another. Regarding the sets of input parameters, the best results were obtained when using real power along with impedance $(P-Z)$, indicating the usefulness of impedance in the development of non-intrusive monitoring models. Overall, the inputs parameters that include real power had a higher success rate than those that include current. It was also achieved that loads with varying power consumption in a small range for each operation state were better identified than those with more variable consumption.

\section{Acknowledgements}

Authors thank the Power System Research Group GISEL of the Universidad del Norte and the Universidad Nacional de Colombia for their financial support to conduct this research. 


\section{REFERENCES}

[1] Gellings, C. W. (2009). The Smart Grid: Enabling Energy Efficiency and Demand Response. Lilburn: The Fairmont Press Inc.

[2] Schneider Electric. (2011). Monitoreo del uso de la energía : el poder de la información. http://www2.schneiderelectric.com/documents/support/white-papers/998-4890 monitoring-energy-use-LAM.pdf

[3] Chang, H. (2010). Load Identification of Non-intrusive Load-monitoring System in Smart Home. WSEAS Trans. Syst., 9(5), 498-510

[4] Najmeddine, H., El Khamlichi Drissi, K., Pasquier, C.; et al. (2008). State of art on load monitoring methods. $2^{\text {nd }}$ IEEE International Conference on Power and Energy (PECon 2008) / Johor Baharu, 1256-1258. https://doi.org/10.1109/PECON.2008.4762669

[5] Hart, G. W., Kern, E. C., \& Schweppe, F. C. (1989). Nonintrusive appliance monitor apparatus. US Patent 4858141, United States, https://www.google.com/patents/US4858141

[6] Hart, G. W. (1992). Nonintrusive appliance load monitoring. Proceedings of the IEEE, 80(12), 1870-1891. https://doi.org/10.1109/5.192069

[7] Patel, S. N., Robertson, T., Kientz, J. A., Reynolds, M. S., \& Abowd G. D. (2007). At the flick of a switch: Detecting and classifying unique electrical events on the residential power line. Proc. $9^{\text {th }}$ Int. Conf. Ubiquitous Comput. / Innsbruck, Austria, 271-288.

[8] Pihala, H. (1998). Non-intrusive appliance load monitoring system based on a modern kWh-meter. VTT Publications.

[9] Bijker, A., Xia, X., \& Zhang, J. (2009). Active Power Residential Non-intrusive Load Monitoring System. IEEE Africon 2009 / Nairobi, Kenya, 1-6. https://doi.org/10.1109/AFRCON.2009.5308244

[10] Farinaccio, L. \& Zmeureanu, R. (1999). Using a pattern recognition approach to disaggregate the total electricity consumption in a house into the major end-uses. Energy and Buildings, 30(3), 245-259. https://doi.org/10.1016/S0378-7788(99)00007-9

[11] Racines, D. L. \& Candelo, J. E. (2014). Non intrusive load identification with power and impedance obtained from smart meters. International Journal of Engineering and Technology, 6(4), 1867-1876.

[12] Srinivasan, D., Ng, W. S., \& Liew, A. C. (2006). NeuralNetwork-Based Signature Recognition for Harmonic Source Identification. IEEE Transactions on Power Delivery, 21(1), 398-405. https://doi.org/10.1109/TPWRD.2005.852370

[13] Zeifman, M. \& Roth, K. (2011). Nonintrusive appliance load monitoring: Review and outlook. IEEE Transactions on Consumer Electronics, 57(1), 76-84. https://doi.org/10.1109/TCE.2011.5735484

[14] Kolter, J. \& Johnson, M. (2011). Redd: A Public Data Set for Energy Disaggregation Research. Proceedings of the SustKDD Workshop on Data Mining Applications in Sustainability / San Diego, CA, USA, 1-6.

[15] Anderson, K., Ocneanu, A., Benitez, D., Carlson, D., Rowe, A., \& Bergés, M. (2012). Blued: a fully labeled public dataset for event-based non-intrusive load monitoring research. Proceedings of the $2^{\text {nd }}$ SustKDD Workshop on Data Mining Applications in Sustainability.

[16] Makonin, S., Popowich, F., Bartram, L., Gill, B., Bajic, I. V. (2013). AMPds : A Public Dataset for Load Disaggregation and Eco-Feedback Research. IEEE Electrical Power \& Energy Conference (EPEC), 1-6. https://doi.org/10.1109/EPEC.2013.6802949

[17] Maasoumy, M., Sanandaji, B. M., Poolla, K., \& Vincentelli A. S. (2013). BERDS-BERkeley EneRgy Disaggregation Data Set. Proceedings of the Workshop on Big Learning at the Conference on Neural Information Processing Systems (NIPS), 1-6.
[18] Rahimi, S., Chan, A. D. C., \& Goubran, R. A. (2012). Nonintrusive load monitoring of electrical devices in health smart homes. IEEE International Instrumentation and Measurement Technology Conference, 2313-2316. https://doi.org/10.1109//2MTC.2012.6229453

[19] Reeg, C. \& Overbye, T. (2010). Algorithm development for non-intrusive load monitoring for verification and diagnostics. North American Power Symposium, 1-5. https://doi.org/10.1109/NAPS.2010.5619600

[20] Cole, A. I. \& Albicki, A. (1998). Data extraction for effective non-intrusive identification of residential power loads. IEEE Instrumentation and Measurement Technology Conference / St. Paul Minnesota, USA, 812-815. https://doi.org/10.1109/IMTC.1998.676838

[21] Anderson, K. D., Berges, M. E., Ocneanu, A., Benitez, D., \& Moura, J. M. F. (2012). Event detection for Non Intrusive load monitoring. IECON 2012 - $38^{\text {th }}$ Annual Conference on IEEE Industrial Electronics Society, 3312-3317. https://doi.org/10.1109/IECON.2012.6389367

[22] Serrano, A., Soria, E., \& Martín, J. (2010). Redes Neuronales Artificiales. Valencia.

[23] Beale, M. H., Hagan, M. T., \& Demuth, H. B. (2013). Neural Network Toolbox ${ }^{\mathrm{TM}}$, User's Guide R 2013 b.

[24] Wilamowski, B. M. \& Hao, Yu. (2010). Improved Computation for Levenberg-Marquardt Training. IEEE Transactions on Neural Networks, 21(6), 930-937. https://doi.org/10.1109/TNN.2010.2045657

[25] Wilamowski, B. M. (2011). How to not get frustrated with neural networks. IEEE International Conference on Industrial Technology, 5-11. https://doi.org/10.1109/ICIT.2011.5754336

[26] Alahmad, M., Hasna, H., \& Sordiashie, E. (2011). Nonintrusive electrical load monitoring and profiling methods for applications in energy management systems. IEEE Long Island Systems, Applications and Technology Conference, 1-6. https://doi.org/10.1109/LISAT.2011.5784233

[27] Chang, H.-H., Chen, K.-L., Tsai, Y.-P., \& Lee, W.-J. (2012). A New Measurement Method for Power Signatures of Nonintrusive Demand Monitoring and Load Identification. IEEE Transactions on Industrial Applications, 48(2), 764771. https://doi.org/10.1109/TIA.2011.2180497

[28] Duan, J., Czarkowski, D., Zabar, Z. (2004). Neural network approach for estimation of load composition. IEEE International Symposium on Circuits and Systems, V-988V-991. ttps://doi.org/10.1109/ISCAS.2004.1329976

\section{Contact information:}

\section{Diana RACINES, MSc}

(Corresponding Author)

Department of Electrical and Electronics Engineering,

Universidad del Norte, Km 5 vía Puerto Colombia, Barranquilla, Colombia

Email: dracines@uninorte.edu.co

\section{John Edwin CANDELO BECERRA, PhD}

Department of Electrical Energy and Automation,

Universidad Nacional de Colombia, Cra. 80 No 65-223, Medellín, Colombia

Email: jecandelob@unal.edu.co

\section{Johny MONTAÑA, PhD}

Department of Electrical Engineering,

Universidad Técnica Federico Santa María, Valparaíso, Chile

Email: johny.montana@usm.cl 